Pacific Journal of Mathematic

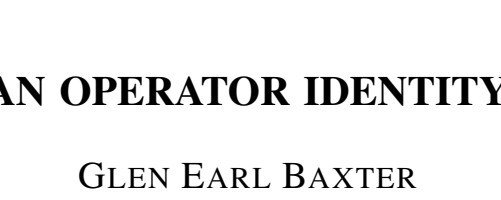




\section{AN OPERATOR IDENTITY}

\section{GLEN BAXTeR}

1. Introduction. Recently, some combinatorial results by Andersen $[1,2]$, Spitzer [5], and others have been applied quite successfully to problems in probability theory. Many of these applications have given rise to results which are entirely analytical in nature. For example, Spitzer used a combinatorial theorem to find the distribution function for the maximum of the partial sums $S_{1}, S_{2}, \cdots, S_{n}$ for a sequence $\left\{X_{k}\right\}$ of independent, identically distributed random variables. His final result is a functional identity,

$$
\sum_{n=0}^{\infty} \varphi_{n}(t) s^{n}=\exp \left\{\sum_{k=1}^{\infty} \frac{s^{k}}{k} \psi_{k}(t)\right\},
$$

where $\varphi_{n}(t)$ is the characteristic function of $\max \left(0, S_{1}, \cdots, S_{n}\right)$ and where $\psi_{k}(t)$ is the characteristic function of $\max \left(0, S_{k}\right)$. One of our purposes in this paper is to generalize (1.1) to an identity involving operators. Our proofs involve more or less analytical methods and thus show that the combinatorial methods hitherto employed can be avoided. We also obtain certain results concerning $\max \left(X_{0}, X_{1}, \cdots, X_{n}\right)$ when $\left\{X_{k}, k \geq 0\right\}$ forms a stationary Markov process.

To illustrate the results we consider a simple example. Let $N$ be an $n \times n$ matrix and let $N^{+}$be the matrix formed from $N$ by replacing with zeros all elements of $N$ which are either on or below the diagonal. Let $N^{-}=N-N^{+}$, and suppose that $N^{+}$and $N^{-}$commute. Now consider the matrix equation

$$
P Q=e^{N}=I+N+N^{2} / 2 !+\cdots
$$

where $P-I$ ( $I$ is the identity matrix) has non-zero terms only above the diagonal and where $Q-I$ has non-zero terms only on or below the diagonal. The properties of $N^{+}$and $N^{-}$imply that

$$
\begin{aligned}
& P=e^{N^{+}}=I+N^{+}+\left(N^{+}\right)^{2} / 2 !+\cdots, \\
& Q=e^{N^{-}}=I+N^{-}+\left(N^{-}\right)^{2} / 2 !+\cdots
\end{aligned}
$$

satisfy (1.2) and have the proper form for $P$ and $Q$. In particular, $\exp \left(N^{+}\right)$has the proper form for $P$ by virtue of the fact that the product of two matrices with non-zero elements only above the diagonal is a

This paper was originally accepted by the Trans. Amer. Math. Soc. Presented to the American Mathematical Society August 28, 1957; received by the editors of the Trans. Amer. Math. Soc., July 29, 1957. This work was supported by the United States Air Force under Contract No. AF18(603)-30 monitored by the Office of Scientific Research. 
matrix of the same type. A similar statement holds for $\exp \left(N^{-}\right)$. It is not hard to see that $P$ and $Q$ are uniquely determined by (1.2). Thus (1.3) is the unique solution of (1.2).

Suppose further that in some neighborhood of $s=0, N=N_{1} s+N_{2} s^{2}$ $+\cdots$, where convergence of the infinite series of $(n \times n)$ matrices is equivalent to convergence of the series of $i j$ th elements for all fixed $i$ and $j$. Relations (1.3) may be rewritten as power series in $s$

$$
P=\sum_{n=0}^{\infty} P_{n} s^{n}, \quad Q=\sum_{n=0}^{\infty} Q_{n} s^{n}
$$

which converge in some neighborhood of $s=0$. It follows from the form of $P$ and $Q$ that $P_{1}, P_{2}, \cdots$ have non-zero elements only above the diagonal while $Q_{1}, Q_{2}, \cdots$ have non-zero elements only on or below the diagonal. Certain problems will lead directly to an equation of the form (1.2) where $P$ and $Q$ have the form (1.4). For example, in one case we will have

$$
P Q=(I-s M)^{-1}=\exp \left\{\sum_{k=1}^{\infty} M^{k} s^{k}\right\}
$$

Under the appropriate commutativity conditions it will follow that

$$
P=\exp \left\{\sum_{k=1}^{\infty}\left(M^{k}\right)^{+} s^{k}\right\}, \quad Q=\exp \left\{\sum_{k=1}^{\infty} \frac{\left(M^{k}\right)^{-}}{k} s^{k}\right\} .
$$

We see later that (1.6) is the operator analogue of Spitzer's identity (1.1) whenever the operator $M$ has a special form.

Equation (1.5) is of particular importance in finding the distribution of $\max \left(X_{0}, X_{1}, \cdots, X_{n}\right)$ when $\left\{X_{k}, k \geq 0\right\}$ is a Markov process with a stationary transition probability matrix $M$. In this case the matrix $M$ in (1.5) is identified (see $\S 4$ ) with the stationary transition probability matrix $M$. Unfortunately, in the general Markov chain, the commutativity conditions which give (1.6) as the solution of (1.5) are not satisfied. Some information can be obtained directly from (1.5).

In the next section we give general definitions and a few preliminary results. The main theorems are proved in $\S 3$ and illustrated in $\S 5$. A probabilistic interpretation of the theorems is contained in $\S 4$.

2. Definitions and preliminaries. Let $L_{0}$ be the space of bounded Baire functions (real-valued and Borel measurable) $f(x)$ on the infinite interval $-\infty<x<\infty$. We will deal with bounded linear operators $M$ defined over $L_{0}$ which have the form

$$
M f \equiv \int_{-\infty}^{\infty} f(y) m(x ; d y)
$$


where $m(x ; A)$ is a function of a real number $x$ and a linear Borel measurable set $A$ such that

(i) for each fixed set $A, m(x ; A)$ is a Baire function of $x$, (2.2) (ii) for each fixed $x, m(x ; A)$ is a signed measure in $A$ on the linear Borel sets.

The norm of the operator $M$ is defined in the usual way in terms of the norm $\|f\|=\max |f(x)|$ in the Banach space $L_{\nu}$. Let $\mu(x ; A)$ and $v(x ; A)$ be, respectivery, the upper variation and the lower variation of the signed measure $m(x ; A)$ (see [4, page 122]) The boundedness of $M$ in (2.1) implies that

$$
\begin{aligned}
\int_{-\infty}^{\infty} & {[\mu(x ; d y)+\nu(x ; d y)] } \\
& \leq \max _{-\infty<x<\infty} \int_{-\infty}^{\infty}[\mu(x ; d y)+\nu(x ; d y)]=\|M\|<\infty .
\end{aligned}
$$

We call $m(x ; A)$ the kernel of the operator $M$. The notation which will be used for integration with respect to a given measure is indicated in (2.1). From now on when we call $M$ a bounded linear operater of the form (2.1), we imply that (2.2) is also satisfied. As a matter of fact, with proper understanding of the notation, (2.2) follows directly from (2.1). If $M_{1}$ and $M_{2}$ are bounded linear operators of the form (2.1) with kernels $m_{1}(x ; A)$ and $m_{2}(x ; A)$, respectively, then $M_{1} M_{2}$ is also of the form (2.1) with kernel

$$
m(x ; A)=\int_{-\infty}^{\infty} m_{2}(y ; A) m_{1}(x ; d y) .
$$

We now let $[x]$ be the greatest integer less than or equal to $x$.

Definition 2.1. Set $B_{n}(x)=\left\{y: y>\left[2^{n} x+1\right] / 2^{n}\right\}$. For any bounded linear operator $M$ of the form $(2.1)$ with kernel $m(x ; A)$, define

$$
m^{+}(x ; A) \equiv \lim _{n \rightarrow \infty} m\left(x ; B_{n}(x) A\right),
$$

and let $M^{+}$be the operator of form (2.1) with kernel $m^{+}(x ; A)$. Finally, set, $M^{-}=M-M^{+}$.

Almost directly from the definition of $M^{+}$follow certain useful facts which we list below. The bounded, linear operators $M, M_{1}, M_{2}$, etc. are all of the form (2.1); $I$ denotes the identity operator, which is also of the form $(2.1)$; and $s, \alpha$, and $\beta$, are real numbers:
(i) $I^{-}=I$,
(ii) $\left(M^{+}\right)^{+}=M^{+}$,
(iii) $\left(M^{-}\right)^{-}=M^{-}$,
(iv) $\left(M_{1}^{+} M_{2}^{+}\right)^{+}=M_{1}^{+} M_{2}^{+}$,
(v) $\left(M_{1}^{-} M_{2}^{-}\right)^{-}=M_{1}^{-} M_{2}^{-}$, 
(vi) $\left\|M^{+}\right\| \leq\|M\|, \quad$ (vii) $\left\|M^{-}\right\| \leq\|M\|$,

(viii) $\left(\alpha M_{1}+\beta M_{2}\right)^{+}=\alpha M_{1}^{+}+\beta M_{2}^{+}$,

(ix) if $M_{0}+M_{2}+\cdots$ is a strongly convergent series of bounded, linear operators of the form (2.1), i.e. if $\left\|M_{n}+\cdots+M_{m}\right\| \rightarrow 0$ as $n, m \rightarrow \infty$, then $T=M_{0}+M_{1}+M_{2}+\cdots$ is of the form (2.1), and $M_{0}^{+}+M_{1}^{+}+M_{2}^{+}+\cdots$ and $M_{0}^{-}+M_{1}^{-}+M_{2}^{-}+\cdots$ are both convergent in the strong sense. Moreover, $T^{+}=$ $M_{0}^{+}+M_{1}^{+}+M_{2}^{+}+\cdots$ and $T^{-}=M_{0}^{-}+M_{1}^{-}+M_{2}^{-}+\cdots$.

We prove only (ix) of (2.6). Let $T_{n}=M_{0}+\cdots+M_{n}$, let $t_{n}(x ; A)$ be the kernel of $T_{n}$, and let $\chi_{A}$ be the characteristic function of a measurable set $A$. If $T=\lim T_{n}$, we note that $\|T\|$ is finite. Now

$$
\left|t_{n}(x ; A)-t_{m}(x ; A)\right|=\left|\left(T_{n}-T_{m}\right) \chi_{A}\right| \leq\left\|T_{n}-T_{m}\right\|,
$$

so that $\lim t_{n}(x ; A)=t(x ; A)$ exists uniformly in $A$. If $A=\Sigma A_{k}$ where the $A_{k}$ are disjoint, then by the Moore double-limit theorem

$$
\sum_{k=1}^{\infty} t\left(x ; A_{k}\right)=\lim _{N \rightarrow \infty} \lim _{n \rightarrow \infty} \sum_{k=1}^{N} t_{n}\left(x ; A_{k}\right)=\lim _{n \rightarrow \infty} t_{n}(x ; A)=t(x ; A) .
$$

This shows that $t(x ; A)$ is a signed measure. Since $T \chi_{A}=t(x ; A)$, a simple argument shows that $t(x ; A)$ is the kernel of $T$. Finally, since $\left\|T^{+}-T_{n}^{+}\right\| \leq\left\|T-T_{n}\right\|$, it follows that $T^{+}=\lim T_{n}^{+}$. In terms of $M_{n}$ this means $T^{+}=M_{0}^{+}+M_{1}^{+}+M_{2}^{+}+\cdots$. A similar argument gives $T^{-}=$ $M_{0}^{-}+M_{1}^{-}+M_{2}^{-}+\cdots$.

It is interesting to note that the proofs of the main theorems will depend only on the facts listed in (2.6). Before proceeding to the next section we mention two special subclasses of operators which have the form (2.1).

Case 1. Let $M=\left(m_{i j}\right)$ be a matrix for which uniformly in $i$

$$
\sum_{(j)}\left|m_{i j}\right|<C
$$

for some constant $C$. For any Borel measurable set $A$ and any real number $x$ define

$$
m(x ; A)= \begin{cases}\sum_{j \in A} m_{i j} & x=i \text { (an integer) } \\ 0 & x \neq[x] .\end{cases}
$$

Condition (2.8) insures the existence of a bounded linear operator of form (2.1) with the kernel $m(x ; A)$ of (2.9). Certainly the operator given by (2.1) in this case and the original matrix $M$ can be identified. In fact, $\mathrm{L}_{0}$ could be replaced here by the class of bounded, doubly infinite sequences $\left\{a_{k}\right\}$, that is $a_{k}=f(k)(-\infty<k<\infty)$ where $f(x) \in L_{0}$. It will 
be convenient whenever possible to think of the matrix $M$ rather than the operator $M$. Note that the matrix $M^{+}$is formed from the matrix $M$ by replacing with zeros all elements of $M$ either on or below the diagonal. Moreover, the matrix $M^{+}$satisfies (2.6).

Case 2. Let $m(x, y)$ be Borel measurable and integrable over the plane and such that for some constant $C$

$$
\int_{-\infty}^{\infty}|m(x, y)| d y<C
$$

uniformly in $x$. For any Borel measurable set $A$ and any real number $x$, define

$$
m(x ; A) \equiv \int_{A} m(x, y) d y
$$

Then, (2.1) gives a bounded, linear operator $M$ which has the form

$$
M \cdot=\int_{-\infty}^{\infty} \cdot m(x, y) d y,
$$

and $M^{+}$becomes simply

$$
M^{+} \cdot=\int_{x}^{\infty} \cdot m(x, y) d y
$$

with a similar formula for $M^{-}$.

3. The theorems. When we say a sequence of operators $\left\{M_{n}\right\}$ converges to an operator $M$, we mean it converges in the strong sense, that is $\left\|M_{n}-M\right\| \rightarrow 0$ as $n$ becomes infinite.

Lemma 3.1. Let $\left\{K_{k}\right\},\left\{P_{k}\right\}$, and $\left\{Q_{k}\right\}, k=1,2,3, \cdots$, be sequences of bounded, linear operators of the form (2.1) for which $P_{k}^{+}=P_{k}$ and $Q_{\bar{k}}^{-}=Q_{k}$. For any $|s|<s_{0}$, let

$$
\begin{aligned}
& P=I+P_{1} s+P_{2} s^{2}+\cdots, \\
& Q=I+Q_{1} s+Q_{2} s^{2}+\cdots, \\
& K=I+K_{1} s+K_{2} s^{2}+\cdots
\end{aligned}
$$

converge. If $P Q=K$ for all $|s|<s_{0}$, then $\left\{P_{k}\right\}$ and $\left\{Q_{k}\right\}$ are uniquely determined by $\left\{K_{k}\right\}$.

Proof. Equating coefficients of like powers of $s$ on the two sides of the equation $P Q=K$ we obtain

$$
\sum_{k=0}^{n} P_{k} Q_{n-k}=K_{n} .
$$


If $P_{1}, P_{2}, \cdots, P_{n-1}$ and $Q_{1}, Q_{2}, \cdots, Q_{n-1}$ have been uniquely determined by $K_{1}, K_{2}, \cdots, K_{n-1}$, then we may write (3.2) as

$$
P_{n}+Q_{n}=J_{n}
$$

where $J_{n}$ is determined uniquely by $K_{1}, K_{2}, \cdots, K_{n}$. Since $P_{n}^{-}=Q_{n}^{+}=0$, we have $P_{n}=J_{n}^{+}$and $Q_{n}=J_{n}^{-}$and the proof follows by induction.

The next theorems give results in the direction of solving equations which involve the operation " +". Later we give a probabilistic interpretation of these equations. As we will see, in certain cases the equations may be solved completely in terms of the known operator $M$.

THEOREM 3.1. Let $M$ be a bounded, linear operator of the form (2.1). Define the sequences $\left\{P_{k}\right\},\left\{Q_{k}\right\},\left\{R_{k}\right\}$, and $\left\{T_{k}\right\}$ by

$$
\begin{aligned}
P_{0} & =Q_{0}=I, & & R_{0}=T_{0}=0, \\
P_{n+1} & =\left(M P_{n}\right)^{+}, & & Q_{n+1}=\left(Q_{n} M\right)^{-}, \\
T_{n+1} & =\left(M P_{n}\right)^{-}, & & R_{n+1}=\left(Q_{n} M\right)^{+},
\end{aligned}
$$

and let the generating functions of these sequences be

$$
\begin{array}{ll}
P=\sum_{n=0}^{\infty} P_{n} s^{n}, & Q=\sum_{n=0}^{\infty} Q_{n} s^{n}, \\
R=\sum_{n=0}^{\infty} R_{n} s^{n}, & T=\sum_{n=0}^{\infty} T_{n} s^{n},
\end{array}
$$

Then, the series' in (3.5) all converge for $|s|<1 /\|M\|$, and, moreover, they are the unique bounded, linear operators of the form (2.1) which satisfy.

$$
\begin{array}{ll}
P=I+s(M P)^{+}, & T=s(M P)^{-}, \\
Q=I+s(Q M)^{-}, & R=s(Q M)^{+} .
\end{array}
$$

Proof. Let $P$ be a bounded, linear operator of the form (2.1) which satisfies the first equation of (3.6). By iteration we may write $P=$ $I+P_{1} s+P_{2} s^{2}+\cdots+P_{n} s^{n}+L_{n}$, where $L_{0}=s(M P)^{+}$and $L_{n}=s\left(M L_{n-1}\right)^{+}$ and where $P_{1}, P_{2}, \cdots, P_{n}$ are determined in (3.4). Property (vi) of (2.6) implies that $\left\|L_{n}\right\| \leq|s|^{n}\|M\|^{n}\|P\|$ which approaches zero as $n$ becomes infinite for all $|s|<1 /\|M\|$. Thus, the solution (if it exists) of the first equation of (3.6) is unique. Let $\left\{P_{k}\right\}$ satisfy the conditions of (3.4). By property (vi) of (2.6), it follows that $\left\|P_{n}\right\| \leq\|M\|^{n}$. For $|s|<1 /\|M\|$, the power series in (3.5) for $P$ converges and by property (ix) of (2.6)

(3.7) $P-I=\sum_{n=0}^{\infty} P_{n+1} s^{n+1}=\sum_{n=0}^{\infty}\left(M P_{n}\right)^{+} s^{n+1}=\left(\sum_{n=0}^{\infty} M P_{n} s^{n+1}\right)^{+}=s(M P)^{+}$.

The proofs of the other parts of the theorem follow similarly. 
Theorem 3.2. Let $|s|<1 /\|M\|$ and let $P$ and $Q$ be the bounded, linear operators of the form (2.1) which satisfy the equations of (3.6). Then,

$$
\begin{aligned}
& P Q=(I-s M)^{-1} \\
& s P^{\prime}=P(Q P-I)^{+}, \quad s Q^{\prime}=(Q P-I)^{-} Q,
\end{aligned}
$$

where' indicates derivative with respect to $s$.

Proof. From (3.6) we find that $\|Q\| \leq 1 /(1-|s|\|M\|)$ and

$$
\|R\| \leq|s|\|M\|\|Q\| \leq|s|\|M\| /(1-|s|\|M\|) .
$$

Thus, for $|s|<(1-|s|\|M\|) /\|M\|$, the operator $(I-R)^{-1}$ is a bounded linear operator of the form (2.1) and has a convergent power series expansion in $s$. But $Q=I-R+s Q M$, or equivalently, $(I-R)^{-1} Q=$ $(I-s M)^{-1}$. Similarly we show that $(I-T)^{-1}$ is a bounded linear operator of the form (2.1) which has a convergent power series expansion in $s$ for $|s|<(1-|s|\|M\|) /\|M\|$, and that $P(I-T)^{-1}=(I-s M)^{-1}$. Applying Lemma 3.1 in the common interval of convergence of $P, Q,(I-T)^{-1}$ and $(I-R)^{-1}$, we deduce that

$$
P=(I-R)^{-1}, \quad Q=(I-T)^{-1} .
$$

and hence that $P Q=(I-s M)^{-1}$. Since $P, Q$, and $(I-s M)^{-1}$ all converge for $|s|<1 /\|M\|$, we have finally $P Q=(I-s M)^{-1}$ for all $|s|<1 /\|M\|$. To show the second half of (3.8), we consider $(P Q)^{\prime}=P^{\prime} Q+P Q^{\prime}=$ $(I-s M)^{-2} M$. It follows that

$$
(P Q)^{2}-s(P Q)^{\prime}=(I-s M)^{-2}(I-s M)=P Q .
$$

Multiplying on the left of (3.10) by $P^{-1}$ and on the right by $Q^{-1}$ (take $|s|<(1-|s|\|M\|) /\|M\|)$ we obtain

$$
Q P-s\left(P^{-1} P^{\prime}+Q^{\prime} Q^{-1}\right)=I .
$$

By properties (iv), (v), and (ix) of (2.6), it is not hard to see that $\left(P^{-1} P^{\prime}\right)^{+}=P^{-1} P^{\prime}$ and $\left(Q^{\prime} Q^{-1}\right)^{-}=Q^{\prime} Q^{-1}$. From (3.11) we find $s P^{\prime}=P(Q P-I)^{+}$ and $s Q^{\prime}=(Q P-I)^{-} Q$. These latter equations can certainly be extended to hold for all $|s|<1 /\|M\|$, and the theorem is proved.

THEOREM 3.3. Let $\left\{a_{k}\right\}$ be a sequence of real numbers such that $a_{1} s+a_{2} s^{2}+a_{3} s^{3}+\cdots$ has a positive radius of convergence. Let $M$ be a bounded, linear operator of the form (2.1) such that $\left(M^{k}\right)^{+} M=M\left(M^{k}\right)^{+}$ for all $k=1,2,3, \cdots$. Then for $|s|$ such that

$$
\sum_{k=1}^{\infty}\left|a_{k}\right|\|M\|^{k}|s|^{k}<1
$$


there is a unique pair of bounded linear operators $P$ and $Q$ of the form (2.1) which satisfy

$$
\begin{aligned}
& P=I+\left[\sum_{k=1}^{\infty}\left(a_{k} M^{k} s^{k}\right) P\right]^{+}, \\
& Q=I+\left[Q \sum_{k=1}^{\infty}\left(a_{k} M^{k} s^{k}\right)\right]^{-} .
\end{aligned}
$$

Moreover, the solution of (3.13) is

$$
\begin{aligned}
& P=\exp \left\{\left[-\log \left(I-\sum_{k=1}^{\infty} a_{k} M^{k} s^{k}\right)\right]^{+}\right\}, \\
& Q=\exp \left\{\left[-\log \left(I-\sum_{k=1}^{\infty} a_{k} M^{k} s^{k}\right)\right]^{-}\right\} .
\end{aligned}
$$

Before proving Theorem 3.3 we mention a result of particular interest which occurs when both Theorems 3.1 and 3.3 apply, i.e. when $a_{1}=1$ and $a_{2}=a_{3}=\cdots=0$.

CoRollary 3.1. Let $M$ be a bounded linear operator of the form (2.1) such that $\left(M^{k}\right)^{+} M=M\left(M^{k}\right)^{+}$for all $k=1,2,3, \cdots$, and let the sequences $\left\{P_{k}\right\}$ and $\left\{Q_{k}\right\}$ be defined as in (3.4). Then, for all $|s|<1 /\|M\|$, the $P$ and $Q$ of (3.5) have the form

$$
P=\exp \left\{\sum_{k=1}^{\infty} \frac{\left(M^{k}\right)^{+}}{k} s^{k}\right\}, \quad Q=\exp \left\{\sum_{k=1}^{\infty} \frac{\left(M^{k}\right)^{-}}{k} s^{k}\right\} .
$$

Proof of Theorem 3.3. Let $|s|$ satisfy the condition of (3.12), and let

$$
\begin{aligned}
& L=\sum_{k=1}^{\infty} a_{k} M^{k} s^{k}, \\
& N=\log \left(I-\sum_{k=1}^{\infty} a_{k} M^{k} s^{k}\right)=\sum_{k=1}^{\infty} L^{k} / k .
\end{aligned}
$$

Both $L$ and $N$ are bounded linear operators of the form (2.1). The commutativity of $\left(M^{k}\right)^{+}$and $M$ together with property (ix) of (2.6) implies that $L^{+} L=L L^{+}$. Again by property (ix) of (2.6) and the second relation of (3.16), we deduce that $N^{+} N=N N^{+}$. In terms of $N$ the first equation in (3.13) may be written in the form

$$
P=I+\left[\left(I-e^{N}\right) P\right]^{+} .
$$

Using that $\left(\exp \left(-N^{+}\right)\right)^{+}=\exp \left(-N^{+}\right)-I$ and that $\left(\exp \left(N^{-}\right)\right)^{+}=0$, it is easy to show by substitution that $P=\exp \left(-N^{+}\right)$is a solution of (3.17). To show that this solution is unique we apply Theorem 3.1, where the operator " $M$ " of Theorem 3.1 is now 


$$
\sum_{k=1}^{\infty} a_{k} M^{k} s^{k}
$$

and the number " $s$ " of Theorem 3.1 is now 1 . In a similar manner we can show that the $Q$ of (3.14) is the unique solution of the second equation in (3.13). This finishes the proof.

Before proceeding into the next section, we point out some implications of the theorems above. In Theorem 3.3, the operators $P, Q, M, M^{+}$, and $M^{-}$all commute. Thus, the order of the factors $Q$ and $M^{k}$ or of $P$ and $M^{k}$ in (3.13) is unimportant. In the $s$ interval determined by (3.12), there is a power series expansion in $s$ for the solutions of (3.13). The coefficients in this power series satisfy

$$
\begin{aligned}
& P_{0}=Q_{0}=I, \\
& P_{n+1}=\left(a_{1} M P_{n}+a_{2} M^{2} P_{n-1}+\cdots+a_{n+1} M^{n+1}\right)^{+}, \\
& Q_{n+1}=\left(a_{1} Q_{n} M+a_{2} Q_{n-1} M^{2}+\cdots+a_{n+1} M^{n+1}\right)^{-} .
\end{aligned}
$$

If the $M$ in Theorem 3.1 is a matrix of finite order, the $P$ and $Q$ of (3.5) can be conveniently evaluated in terms of subdeterminants of the matrix $I-s M$ (See example $3, \S 5$ ).

4. Probabilistic interpretation. In this section we give a probabilistic interpretation of the sequences $\left\{P_{k}\right\},\left\{Q_{k}\right\},\left\{R_{k}\right\}$, and $\left\{T_{k}\right\}$ of Theorem 3.1. Let $m(x ; A)$ be a function of a real number $x$ and a linear Borel measurable set $A$ such that

(i) for each fixed set $A, m(x ; A)$ is a Baire function of $x$,

(ii) for each fixed $x, m(x ; A)$ is a probability measure in $A$ on the linear Borel measurable sets.

Let $\left\{X_{k}, k \geq 0\right\}$ be a stationary Markov process for which $m(x ; A)=$ $P\left\{X_{k+1} \in A \mid X_{k}=x\right\}$ is defined and satisfies the conditions of (4.1) (see [3, pp. 18, 26-27]). We deal here only with processes of this type. By (2.1) and (2.3) each Markov process under consideration has associated with it a bounded linear operator $M$, with $\|M\|=1$. We call this the transition probability operator of the process.

Two subcases of special interest may be mentioned. The first one is that of a discrete Markov chain (countable state space). In this case the transition probabilities form a matrix $M=\left(m_{i j}\right)$. The connection between the matrix $M$ and the function $m(x ; A)$ has already been discussed in $\S 2$, case 1 . The second type process of interest is the one for which the joint distributions have densities. In this latter case, there exists a transition probability density function $m(x, y)$, and the connection with $m(x ; A)$ is given in $\S 2$, case 2 .

For convenience in stating the next theorem we introduce a random variable $L_{n}$. 
$L_{n}:$ the index $k(=0,1,2, \cdots)$ for which $\max \left(X_{0}, X_{1}, \cdots, X_{n}\right)$ $=X_{k}$ and $\max \left(X_{0}, X_{1}, \cdots, X_{k-1}\right)<X_{k}$.

Note in particular the meaning of the statements $L_{n}=n$ and $L_{n}=0$. In Theorem 4.1 and thereafter we will have occasion to refer to the kernel associated with a given operator of the form (2.1). If the operator is denoted by some capital letter, the kernel will be denoted by the corresponding small letter.

Theorem 4.1. Let $\left\{X_{k}, k \geq 0\right\}$ be a stationary Markov process with transition probability operator $M$, and let $\left\{P_{k}\right\},\left\{Q_{k}\right\},\left\{R_{k}\right\}$ and $\left\{T_{k}\right\}$ be defined as in (3.4). Then, if the right hand members of (4.3) are defined and satisfy (2.2), we have

$$
\begin{aligned}
& p_{n}(x ; A)=P\left\{L_{n}=n, X_{n} \in A \mid X_{0}=x\right\}, \\
& q_{n}(x ; A)=P\left\{L_{n}=0, X_{n} \in A \mid X_{0}=x\right\}, \\
& r_{n}(x ; A)=P\left\{L_{n}=n, L_{n-1}=0, X_{n} \in A \mid X_{0}=x\right\}, \\
& t_{n}(x ; A)=P\left\{L_{n}=0, \max \left(X_{1}, \cdots, X_{n-1}\right)<X_{n}, X_{n} \in A \mid X_{0}=x\right\} .
\end{aligned}
$$

Proof. We prove only the first one of the relations in (4.3). Our proof is by induction. Since $P_{0}=I$, it follows that

$$
p_{0}(x ; A)=P\left\{X_{0} \in A \mid X_{0}=x\right\}=\left\{\begin{array}{ll}
1 & x \in A \\
0 & x \notin A
\end{array} .\right.
$$

Now assume the first relation of (4.3) is true for the case $n$ and set $B_{N}(x)=\left\{y: y>\left[2^{N} x+1\right] / 2^{N}\right\}$ for $N=1,2,3, \cdots$. Then,

$$
\begin{aligned}
& P\left\{L_{n+1}=n+1, X_{n+1} \in B_{N}(x) A \mid X_{0}=x\right\} \\
& =\int_{-\infty}^{\infty} P\left\{\max \left(X_{1}, \cdots, X_{n}\right)<X_{n+1}, X_{n+1} \in B_{N}(x) A \mid X_{1}=z\right\} \\
& \text { - } P\left\{X_{1} \in d z \mid X_{0}=x\right\} \\
& =\int_{-\infty}^{\infty}\left\{L_{n}=n, X_{n} \in B_{N}(x) A \mid X_{0}=z\right\} P\left\{X_{1} \in d z \mid X_{0}=x\right\} \\
& =\int_{-\infty}^{\infty} p_{n}\left(z ; B_{N}(x) A\right) m(x ; d z) \text {. }
\end{aligned}
$$

From (2.4) we see that the last term of (4.5) is the kernel of $M P_{n}$ evaluated at $x$ and $B_{N}(x) A$. Set $A_{x}=A \cap(x, \infty)$, and note that for any $n>0$,

$$
P\left\{L_{n}=n, X \in A \mid X_{0}=x\right\}=P\left\{L_{n}=n, X_{n} \in A_{x} \mid X_{0}=x\right\} .
$$

Thus, by Definition 2.1 and (4.5) 


$$
\begin{aligned}
p_{n+1}(x ; A) & =\lim _{N \rightarrow \infty} \int_{-\infty}^{\infty} p_{n}\left(z ; B_{N}(x) A, m(x ; d z)\right. \\
& =\lim _{N \rightarrow \infty} P\left\{L_{n+1}=n+1, X_{n+1} \in B_{N}(x) A \mid X_{0}=x\right\} \\
& =P\left\{L_{n+1}=n+1, X_{n+1} \in A_{x} \mid X_{0}=x\right\} \\
& =P\left\{L_{n+1}=n+1, X_{n+1} \in A \mid X_{0}=x\right\},
\end{aligned}
$$

and the proof follows by induction.

Combining the first and second of the relations in (4.3) we get certain additional information about $\max \left(X_{0}, \cdots, X_{n}\right)$. In fact, we can evaluate the generating function

$$
\sum_{n=0}^{\infty} P\left\{\max \left(X_{\mathfrak{v}}, \cdots, X_{n}\right) \in A \mid X_{0}=x\right\} s^{n}
$$

in terms of the kernels of $P$ and $Q$. Let $S=(-\infty, \infty)$. Then, by Theorem 4.1

$$
\begin{aligned}
& P\left\{\max \left(X_{0}, \cdots, X_{n}\right) \in A \mid X_{0}=x\right\} \\
& \quad=\sum_{k=0}^{n} P\left\{L_{n}=k, \max \left(X_{0}, \cdots, X_{n}\right) \in A \mid X_{\mathrm{v}}=x\right\} \\
& \quad=\sum_{k=0}^{n} \int_{A} P\left\{L_{n-k}=0 \mid X_{0}=y\right\} P\left\{L_{k}=k, X_{k} \in d y \mid X_{0}=x\right\} \\
& \quad=\sum_{k=0}^{n} \int_{A} q_{n-k}(y ; S) p_{k}(x ; d y) .
\end{aligned}
$$

Multiplying through (4.7) by $s^{n}$ and summing over $s=0,1,2, \cdots$ we obtain

$$
\int_{A} q(y ; S) p(x ; d y)=\sum_{n=0}^{\infty} P\left\{\max \left(X_{0}, \cdots, X_{n}\right) \in A \mid X_{0}=x\right\} s^{n} .
$$

Relation (4.8) takes on a particularly simple form if $q(y ; S)$ is independent of $y$ (See example $2, \S 5$ ). In fact, in this special case we have the following Corollary to Theorem 4.1:

CoROLlary 4.1. Let $\left\{X_{k}, k \geq 0\right\}$ be a stationary Markov process with transition probability operator $M$ and let $P$ and $Q$ be defined as in Theorem 3.1. Furthermore, let $q(x ; A)$ be the kernel of $Q$, and let $\Phi$ be the bounded, linear operator of the form (2.1) determined by

$$
\varphi(x ; A)=\sum_{n=0}^{\infty} P\left\{\max \left(X_{0}, \cdots, X_{n}\right) \in A \mid X_{0}=x\right\} s^{n} .
$$

Then, if $q(x ; S)=q$ is independent of $x$,

$$
\Phi=q P .
$$

Relation (4.10) is an operator analogue of Spitzer's identity (1.1). 
5. Examples. We now give applications of the theorems to some particular examples.

EXAMPle 1. Let the operator of form (2.1) be (See case $1, \S 2$ )

$$
M=\left[\begin{array}{ccc}
a & 0 & b \\
(a-c) d / b & c & d \\
0 & 0 & a
\end{array}\right],
$$

so that for $k=1,2,3, \cdots$

$$
M^{k}=\left[\begin{array}{ccc}
a & 0 & k a^{k-1} b \\
\left(a^{k}-c^{k}\right) d / b & c^{k} & k a^{k-1} d \\
0 & 0 & a^{k}
\end{array}\right] .
$$

It is not hard to see that $\left(M^{k}\right)^{+} M=M\left(M^{k}\right)^{+}$in this case so Corollary 3.1 applies here. The solution of $P=I+s(M P)^{+}$for $|s|<1 /\|M\|<$ $1 /|a|$ is

$$
\begin{aligned}
P & =\exp \left\{\sum_{k=1}^{\infty} \frac{s^{k}}{k}\left(M^{k}\right)^{+}\right\}=\exp \left\{\sum_{k=1}^{\infty} s^{k}\left[\begin{array}{ccc}
0 & 0 & a^{k-1} b \\
0 & 0 & a^{k-1} d \\
0 & 0 & 0
\end{array}\right]\right) \\
& =\exp \left(\left[\begin{array}{ccc}
0 & 0 & b s /(1-a s) \\
0 & 0 & d s /(1-a s) \\
0 & 0 & 0
\end{array}\right]\right\}=\left[\begin{array}{ccc}
1 & 0 & b s /(1-a s) \\
0 & 1 & d s /(1-a s) \\
0 & 0 & 1
\end{array}\right] .
\end{aligned}
$$

In a similar manner it follows that the solution of $Q=I+s(Q M)^{-}$for $|s|<1 /\|M\|<1 / \min (|a|,|c|)$ is

$$
Q=\left[\begin{array}{ccc}
1 /(1-a s) & 0 & 0 \\
(a-c) d s / b(1-a s)(1-c s) & 1 /(1-c s) & 0 \\
0 & 0 & 1 /(1-a s)
\end{array}\right] .
$$

These solutions are easily checked by substitution.

ExAMPle 2. Let $\left\{X_{k}\right\}(k=1,2,3, \cdots)$ be a sequence of independent, identically distributed random variables with a common density function $f(x)$, and let $S_{n}=X_{1}+\cdots+X_{n}$. If $T_{0}$ is any random variable independent of $\left\{X_{k}\right\}$, and if we set $T_{n}=S_{n}+T_{0}(n=1,2,3, \cdots)$, then $\left\{T_{n}, n \geq 0\right\}$ is a stationary Markov process with transition probability

$$
m(x ; A) \equiv P\left\{T_{k+1} \in A \mid T_{k}=x\right\}=\int_{A} f(y-x) d y .
$$

The conditions (4.1) are satisfied by $m(x ; A)$ (as well as by the right hand members of (4.3)) in this case so we so may talk about the 
transition probability operator $M$ associated with $\left\{T_{n}, n \geq 0\right\}$. This operator has the form

$$
M \cdot=\int_{-\infty}^{\infty} \cdot f(y-x) d y .
$$

Using (2.4) and (5.6) it is not hard to deduce that $M^{k}$ also has a kernel with a density. In fact,

$$
M^{k} \cdot=\int_{-\infty}^{\infty} \cdot f_{k}(y-x) d y,
$$

where $f_{k}(x)$ is the $k$-fold convolution of $f(x)$ with itself.

By (5.6), (5.7), and (2.4) we see that the kernel of $\left(M^{k}\right)^{+} M$ has a density of the form

$$
\int_{-\infty}^{\infty} f(y-w) f_{k}^{+}(w-x) d w=\int_{x}^{\infty} f(y-w) f_{k}(w-x) d w .
$$

We now make the change of variable $z=y+x-w$ in the second integral of (5.8) to get

$$
\int_{y}^{\infty} f_{k}(y-z) f(z-x) d z=\int_{-\infty}^{\infty} f_{k}^{+}(y-z) f(z-x) d z .
$$

The second term of (5.9) is the density of the kernel of $M\left(M^{k}\right)^{+}$. Thus, $\left(M^{k}\right)^{+} M=M\left(M^{k}\right)^{+}$in this case and Corollary 3.1 applies. If $P$ and $Q$ are as defined in Theorem 3.1, then for $|s|<1$ (that is $\|M\|=1$ )

$$
P=\exp \left\{\sum_{k=1}^{\infty} \frac{\left(M^{k}\right)^{+} s^{k}}{k}\right\}, \quad Q=\exp \left\{\sum_{k=1}^{\infty} \frac{\left(M^{k}\right)^{-} s^{k}}{k}\right\} .
$$

Since $\left(M^{k}\right)^{-}$has a kernel with a density of the form $f_{k}(y-x)$, we deduce that $Q$ must have a kernel with a density of the form $q(y-x)$. This means

$$
q(x ; S)=\int_{-\infty}^{\infty} q(y-x) d y=\exp \left[\sum_{k=1}^{\infty} \frac{P\left\{S_{k} \leq 0\right\}}{k} s_{k}\right]
$$

is independent of $x$ and Corollary 4.1 applies. Spitzer's identity (1.1) is found in this case from (4.10) by operating with each side on the function $g(y)=\exp (i t y)$. In fact, in the notation of (1.1)

$$
\begin{aligned}
\Phi g & =e^{i t x} \sum_{n=0}^{\infty} \int_{-\infty}^{\infty} e^{i t(y-x)} P\left\{\max \left(T_{0}, \cdots, T_{n}\right) \in d y \mid T_{0}=x\right\} s^{n} \\
& =e^{i t x} \sum_{n=0}^{\infty} \int_{-\infty}^{\infty} e^{i t y} P\left\{\max \left(0, S_{1}, \cdots, S_{n}\right) \in d y\right\} s^{n} \\
& =e^{i t x} \sum_{n=0}^{\infty} \phi_{n}(t) s^{n} .
\end{aligned}
$$


Now in the special case of the exponential function $g(y)=e^{i t y}$,

$$
\left(M^{k}\right)^{+}\left(M^{n}\right)^{+} g e^{-i t x}=\left[\left(M^{k}\right)^{+} g e^{-i t x}\right]\left[\left(M^{n}\right)^{+} g e^{-i t x}\right] .
$$

From (6.10), we find ${ }^{1}$

$$
\begin{aligned}
P g & =e^{i t x} \exp \left\{\sum_{k=1}^{\infty} \frac{\left(M^{k}\right)^{+} g e^{-i t x}}{k} s^{n}\right\} \\
& =e^{i t x} \exp \left[\sum_{k=1}^{\infty} \frac{s^{k}}{k} \int_{0}^{\infty} e^{i t y} P\left\{S_{k} \in d y\right\}\right] .
\end{aligned}
$$

Putting (5.11), (5.12), and (5.14) into (4.10), it follows that

$$
\sum_{n=1}^{\infty} \varphi_{n}(t) s^{n}=\exp \left[\sum_{k=1}^{\infty} \frac{s^{k}}{k} \int_{0}^{\infty} e^{i t y} P\left\{\max \left(0, S_{k}\right) \in d y\right\}\right] .
$$

In passing we note that the existence of a density is convenient but not necessary for the derivation of (1.1) from (4.10). In general, we can replace $(5.5)$ by

$$
m(x ; A)=P\left\{\left(X_{1}+x\right) \in A\right\},
$$

which is Borel measurable in $x$ for each fixed set $A$. The conditions (4.1) are satisfied and the derivation continues in the obvious manner.

EXAMPLE 3. Let $M$ be a matrix of finite order. We denote by $D_{k}$ the subdeterminant formed from the determinant of $I-s M$ by crossing out all but the first $k$ rows and columns. Moreover, $D_{k}(i ; j)(1 \leq i, j \leq k)$ will denote the cofactor of the $i j$ th element in $D_{k}$. Finally, for any matrix $N$, let $N(k)$ denote the matrix formed from $N$ by crossing out all but the first $k$ rows and columns.

Let $\left\{P_{n}\right\},\left\{Q_{n}\right\}, P=\left(p_{i j}\right)$, and $Q=\left(q_{i j}\right)$ denote the matrices defined by (3.4) and (3.5) when Theorem 3.1 is applied to $M$. We may also apply Theorem 3.1 to $M(k)$. It is not hard to show by induction that $\left\{P_{n}(k)\right\},\left\{Q_{n}(k)\right\}, P(k)$, and $Q(k)$ are the matrices defined by (3.4) and (3.5) when Theorem 3.1 is applied to $M(k)$. Thus, by (3.8)

$$
P(k) Q(k)=[I(k)-s M(k)]^{-1} \text {. }
$$

Equating elements of the last row (the $k$ th row) in the matrix product of (5.16), we find

$$
q_{k j}=D_{k}(j ; k) / D_{k}, \quad j=1,2, \cdots, k .
$$

Using (5.17) and the elements of the last column of the product in (5.16), it follows that

$$
p_{i k}=D_{k}(k ; i) / D_{k-1}, \quad i=1,2, \cdots, k .
$$

1 The referee points out that (5.14) holds if and only if $g$ is the exponential function. 
Let $M$ be the transition probability matrix of a stationary Markov chain $\left\{X_{k}, k \geq 0\right\}$ with states $\alpha_{1}<\alpha_{2}<\cdots<\alpha_{N}$. From (4.3), we find

$$
\begin{aligned}
& P\left\{L_{n}=n, X_{n}=\alpha_{j} \mid X_{0}=\alpha_{i}\right\}=D_{j}(j ; i) / D_{j-1}, \quad(i \leq j), \\
& P\left\{L_{n}=0, X_{n}=\alpha_{j} \mid X_{0}=\alpha_{i}\right\}=D_{i}(j ; i) / D_{i}, \quad(i \leq j) \text {. }
\end{aligned}
$$

\section{REFERENCES}

1. E. Sparre Andersen, On the fluctuations of sums of random variables, Math. Scand. 1 (1953), 263-285.

2. - On the fluctuations of sums of random variables II, Math. Scand. 2 (1954), 195-223.

3. J. L. Doob, Stochastic process, New York 1953.

4. P. R. Halmos, Measure theory, New York 1958.

5. Frank Spitzer, A combinatorial lemma and its application to probability theory, Trans. Amer. Math. Soc. 82 (1956), 323-339.

UNIVERSITY OF MINNESOTA 



\section{PACIFIC JOURNAL OF MATHEMATICS}

\section{EDITORS}

\section{David Gilbarg}

Stanford University

Stanford, California

\section{R. A. Beaumont}

University of Washington

Seattle 5, Washington

\author{
A. L. Whiteman
}

University of Southern California Los Angeles 7, California

E. G. Straus

University of California

Los Angeles 24, California

\section{ASSOCIATE EDITORS}

\author{
E. F. BECKENBACH \\ C. E. BURGESS \\ M. HALL \\ E. HEWITT
}
A. HORN
V. GANAPATHY IYER
R. D. JAMES
M. S. KNEBELMAN
L. NACHBIN
I. NIVEN
T. G. OSTROM
H. L. ROYDEN

M. M. SCHIFFER

G. SZEKERES

F. WOLF

K. YOSIDA

\section{SUPPORTING INSTITUTIONS}

\author{
UNIVERSITY OF BRITISH COLUMBIA \\ CALIFORNIA INSTITUTE OF TECHNOLOGY \\ UNIVERSITY OF CALIFORNIA \\ MONTANA STATE UNIVERSITY \\ UNIVERSITY OF NEVADA \\ OREGON STATE COLLEGE \\ UNIVERSITY OF OREGON \\ OSAKA UNIVERSITY \\ UNIVERSITY OF SOUTHERN CALIFORNIA
}

\author{
STANFORD UNIVERSITY \\ UNIVERSITY OF TOKYO \\ UNIVERSITY OF UTAH \\ WASHINGTON STATE COLLEGE \\ UNIVERSITY OF WASHINGTON \\ * * * * \\ AMERICAN MATHEMATICAL SOCIETY \\ CALIFORNIA RESEARCH CORPORATION \\ HUGHES AIRCRAFT COMPANY \\ THE RAMO-WOOLDRIDGE CORPORATION
}

Mathematical papers intended for publication in the Pacific Journal of Mathematics should be typewritten (double spaced), and the author should keep a complete copy. Manuscripts may be sent to any one of the four editors. All other communications to the editors should be addressed to the managing editor, E. G. Straus at the University of California, Los Angeles 24, California.

50 reprints per author of each article are furnished free of charge; additional copies may be obtained at cost in multiples of 50 .

The Pacific Journal of Mathematics is published quarterly, in March, June, September, and December. The price per volume (4 numbers) is $\$ 12.00$; single issues, $\$ 3.50$. Back numbers are available. Special price to individual faculty members of supporting institutions and to individual members of the American Mathematical Society: $\$ 4.00$ per volume; single issues, $\$ 1.25$.

Subscriptions, orders for back numbers, and changes of address should be sent to Pacific Journal of Mathematics, 2120 Oxford Street, Berkeley 4, California.

Printed at Kokusai Bunken Insatsusha (International Academic Printing Co., Ltd.), No. 6, 2-chome, Fujimi-cho, Chiyoda-ku, Tokyo, Japan.

PUBLISHED BY PACIFIC JOURNAL OF MATHEMATICS, A NON-PROFIT CORPORATION

The Supporting Institutions listed above contribute to the cost of publication of this Journal, but they are not owners or publishers and have no responsibility for its content or policies. 


\section{Pacific Journal of Mathematics}

\section{Vol. 8, No. $4 \quad$ June, 1958}

Richard Arens, The maximal ideals of certain functions algebras ........ 641

Glen Earl Baxter, An operator identity ........................... 649

Robert James Blattner, Automorphic group representations ........... 665

Steve Jerome Bryant, Isomorphism order for Abelian groups ............ 679

Charles W. Curtis, Modules whose annihilators are direct summands...... 685

Wilbur Eugene Deskins, On the radical of a group algebra ............ 693

Jacob Feldman, Equivalence and perpendicularity of Gaussian

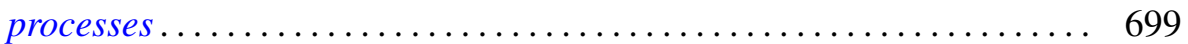

Marion K. Fort, Jr. and G. A. Hedlund, Minimal coverings of pairs by triples....................................... 709

I. S. Gál, On the theory of $(m, n)$-compact topological spaces ......... 721

David Gale and Oliver Gross, A note on polynomial and separable games........................................ 735

Frank Harary, On the number of bi-colored graphs ............... 743

Bruno Harris, Centralizers in Jordan algebras ................... 757

Martin Jurchescu, Modulus of a boundary component ............... 791

Hewitt Kenyon and A. P. Morse, Runs . . . . . . . . . . . . . . . . . . . . . . 811

Burnett C. Meyer and H. D. Sprinkle, Two nonseparable complete metric

spaces defined on $[0,1] \ldots \ldots \ldots \ldots \ldots \ldots \ldots \ldots \ldots \ldots \ldots . \ldots . \ldots . \ldots . \ldots 25$

M. S. Robertson, Cesàro partial sums of harmonic series expansions...... 829

John L. Selfridge and Ernst Gabor Straus, On the determination of numbers by their sums of a fixed order ........................ 847

Annette Sinclair, A general solution for a class of approximation

problems .................................

George Szekeres and Amnon Jakimovski, $(C, \infty)$ and $(H, \infty)$ methods of summation...................................... 867

Hale Trotter, Approximation of semi-groups of operators. ............. 887

L. E. Ward, A fixed point theorem for multi-valued functions ........... 921

Roy Edwin Wild, On the number of lattice points in $x^{t}+y^{t}=n^{t / 2} \ldots \ldots .929$ 